\title{
Intervenção fisioterapêutica em paciente com botulismo em um hospital de alta complexidade
}

\author{
Physiotherapeutic intervention in a patient with botulism in a high complexity hospital
}

Intervención fisioterapéutica en paciente con botulismo en hospital de alta complejidad

Tatielle Eliandra da Silva ${ }^{1 *}$, Leiri Bonet ${ }^{2}$, Geovana Dombrowski Andrade ${ }^{1}$, Samuel Amorim Dias ${ }^{1}$.

\begin{abstract}
RESUMO
Objetivo: Relatar o tratamento fisioterápico em uma paciente com botulismo no interior de RO, descrevendo as alterações funcionais e o tratamento fisioterapêutico aplicado. Detalhamentos de caso: Paciente do gênero feminino, 21 anos, iniciou com mal estar geral após um almoço/jantar em família, onde segundo familiares, ingeriu carne assada e maionese com creme caseiro (ovos e milho), buscou ajuda médica onde o diagnóstico clínico foi suspeita de Salmonelose ou botulismo, tendo em vista que o tratamento para botulismo deve ser iniciado antes das 48 horas, foi administrado soro antibotulínico e iniciado antibioticoterapia. Ao todo foram 84 dias de internação na UTI, gerando fraqueza muscular generalizada, rigidez articular de MMII, imobilismo, uso prolongado de VMI, desmame difícil e pneumonia. Durante esse período, foi realizado atendimento fisioterapêutico intensivo, onde foram traçadas metas diárias e uso de planos terapêuticos para a melhor evolução da paciente. Considerações finais: O botulismo alimentar consiste em uma patologia grave e no Brasil é considerada emergência médica e de notificação compulsória. Diante das alterações funcionais a qual a paciente apresentou 0 tratamento fisioterapêutico, foi de suma importância para a evolução e melhora desde a internação até a alta hospitalar.
\end{abstract}

Palavras-chave: Botulismo, Cuidados intensivos, Intoxicação alimentar, Fisioterapia.

\begin{abstract}
Objective: To report the physiotherapy treatment in a patient with botulism in an inland city of RO, describing the functional changes and the applied physiotherapy treatment. Case details: Female patient, 21 years old, started with general malaise after a family lunch/dinner where, according to family members, she ate roasted meat and mayonnaise with homemade cream (eggs and corn), she sought medical help where the clinical diagnosis was suspected of Salmonellosis or botulism, considering that the treatment for botulism must be started before 48 hours, therapeutic antitoxin was administered and antibiotic therapy was started. Altogether, there were 84 days in the ICU, generating generalized muscle weakness, lower limb joint stiffness, immobilization, prolonged use of invasive mechanical ventilation, difficult weaning and pneumonia. During this period, intensive physical therapy was performed, where daily goals were set and the use of therapeutic plans for the better evolution of the patient. Final considerations: Food botulism is a serious pathology and in Brazil it is considered a medical emergency and mandatory reporting. In view of the functional changes that the patient presented, physical therapy treatment was of paramount importance for the evolution and improvement from hospitalization to hospital discharge.
\end{abstract}

Key words: Botulism, Critical care, Foodborne diseases, Physical therapy specialty.

\section{RESUMEN}

Objetivo: Informar el tratamiento de fisioterapia en un paciente con botulismo dentro del RO, describiendo los cambios funcionales y el tratamiento de fisioterapia aplicado. Detalles del caso: Paciente de sexo femenino, 21 años, inició con malestar general luego de un almuerzo/cena familiar donde, según familiares, comió carne asada y mayonesa con crema casera (huevos y maíz), buscó atención médica donde el diagnóstico clínico fue sospechado de Salmonelosis o botulismo, considerando que el tratamiento del botulismo debe iniciarse antes de las 48 horas, se administró suero de antibotulina y se inició antibioterapia. En total fueron 84 días en UCI, generando debilidad muscular generalizada, rigidez de miembros inferiores, inmovilización, uso prolongado de ventilación mecánica invasiva, destete difícil y neumonía. Durante este período se realizó fisioterapia intensiva, donde se establecieron metas diarias y el uso de planes terapéuticos para la mejor evolución del paciente. Consideraciones finales: El botulismo alimentario es una afección grave y en Brasil se considera una emergencia médica y un informe obligatorio. Ante los cambios funcionales que presentaba el paciente, el tratamiento fisioterapéutico fue de suma importancia para la evolución y mejoría desde la hospitalización hasta el alta hospitalaria.

Palabras clave: Botulismo, Cuidados intensivos, Enfermedades transmitidas por los alimentos, Fisioterapia.

\footnotetext{
${ }^{1}$ Hospital Regional de Cacoal (HRC), Cacoal - RO. *E-mail: tataeliandra@outlook.com

${ }^{2}$ Associação Brasileira de Fisioterapia Cardiorrespiratória e Fisioterapia em Terapia Intensiva (ASSOBRAFIR), Cacoal - RO.
} 


\section{INTRODUÇÃO}

O termo botulismo é utilizado para designar a intoxicação alimentar pelo clostridium botulinum, nome proveniente do latim onde botulus significa salsicha, alimento envolvido nos primeiros casos de botulismo descrito cientificamente na Alemanha, no século XVIII. É uma doença grave, não contagiosa e de alta letalidade, que para minimizar o risco de morte e sequelas é essencial que o diagnóstico e tratamento sejam instituídos precocemente (BARBOZA MMO, et al., 2011; BRASIL, 2006)

A preparação de conservas caseiras constitui o maior risco de contaminação, em razão dos procedimentos inadequados para o seu preparo, deixando o ambiente propício para a bactéria. Além das conservas caseiras, alimentos adquiridos em estabelecimentos clandestinos ou cujas latas estejam danificadas ou estufadas devem ser imediatamente rejeitados e destruídos, pois estes são considerados os maiores responsáveis pelos surtos de intoxicação (CERESER ND, et al., 2008).

No botulismo alimentar a toxina é absorvida pelo trato gastrintestinal e disseminado por via sanguínea até as terminações nervosas, bloqueando a transmissão de acetilcolina, molécula responsável pela passagem de impulso nervoso dos neurônios para as células musculares, ocasionando falha de transmissão de impulsos das fibras nervosas resultando em paralisia flácida. O dano causado é permanente e necessita de formação de novas terminações neuromusculares, ocasionando um período prolongado de internação. Cerca de $30 \%$ a $50 \%$ evoluem para o uso de ventilação mecânica invasiva (VMI) e monitorização contínua em unidade de terapia intensiva (UTI) (BRASIL, 2006).

O diagnóstico é obtido através de exames laboratoriais com o método de pesquisa da toxina botulínica no sangue, fezes, lavados gástricos ou alimentos. O resultado deste exame caracteriza o tipo de botulismo e 0 tipo de toxina encontrada no organismo. Em todos os tipos o quadro clínico é basicamente o mesmo. Inicialmente os sinais e sintomas são gastrointestinais como náuseas, vômitos, diarreias e dor intestinal. 0 quadro neurológico se instala através de manifestações de cefaleias, vertigens e a paralisia se instala de modo descendente (ZATTI CA, 2013; FIGUEIREDO MMA, et al., 2006).

O protocolo de tratamento é composto por suporte clínico intensivo, sendo o tratamento específico a administração do soro antibotulínico o qual age nas toxinas que ainda não atingiram as células nervosas, porém é necessário que sua administração seja o mais precoce possível ou num prazo de até 7 dias de início dos sintomas (BRASIL, 2017; CARDOSO T, et al., 2004).

Diante deste caso considerado raro, o objetivo principal deste estudo é relatar o tratamento fisioterapêutico aplicado durante todo o período de internação, descrevendo e apontando quais foram as principais alterações funcionais, as intervenções fisioterapêuticas aplicadas e ainda relacionando os resultados alcançados após as intervenções, condutas e protocolos aplicados.

\section{DETALHAMENTO DO CASO}

J. S. O. N., 21 anos, feminino natural da zona rural de um município no interior do Estado de Rondônia (RO). Em 10/02/2019 iniciou com mal-estar geral e diplopia e por volta das vinte e duas horas buscou assistência médica junto ao hospital municipal. Evoluiu com disartria, disfagia, insuficiência respiratória sendo necessário iniciar suporte de O2 através de máscara de Venturi 50\%. Na madrugada do dia 11/02/2019 foi transferida para um hospital de emergência e urgência. Admitida às 06h20min da manhã, consciente, em respiração espontânea com suporte de oxigênio através de máscara de Venturi 50\%. Minutos depois, apresentou Rebaixamento de Nível de Consciência (RNC) e foi submetida à Intubação Orotraqueal (IOT). Às 18:00 horas, do mesmo dia, foi transferida para uma UTI Adulto, sendo iniciado uso de droga vasoativa.

Por volta de 9 a 10 horas antes do início dos primeiros sintomas a paciente participou de um almoço em família, onde ingeriu carne assada e maionese com creme caseiro (ovo e milho) no almoço e no jantar, foi relatado pelo acompanhante que demais membros da família que participaram desse almoço também apresentaram os mesmos sintomas.

O médico que realizou o primeiro atendimento levantou as seguintes hipóteses diagnósticas: salmonelose ou botulismo, sendo primordial para a eficácia e bom prognóstico no tratamento da paciente, pois nas primeiras 24 horas foi iniciado tratamento medicamentoso com os antimicrobianos Ciprofloxacino 
Metronidazol e a toxina antibotulínica (CRUZ M, 2019). A confirmação diagnóstica de botulismo ocorreu por meio de exame laboratorial que chegou no dia 08/03/19, confirmando o botulismo do tipo $A$, exame este que foi realizado por um laboratório em São Paulo.

Foi realizada traqueostomia (TQT) no oitavo dia de internação e permaneceu por 90 dias. Evoluiu com instabilidade hemodinâmica, uso de drogas vasoativas DVA, chegando a $60 \mathrm{ml} / \mathrm{hm}$ de noradrenalina, leucocitose, diminuição da diurese, acidose metabólica, e elevação das escores renais, sendo necessário, em 07/03/2019, a realização de hemodiálise. Dia 09/03/2019 apresentou Parada Cardiorrespiratória (PCR), foi realizado um ciclo de 2 minutos de reanimação cardiopulmonar (RCP). Também necessitou de alimentação parenteral total (NPT) por 8 dias, devido à gravidade que a paciente evoluiu, hemotransfusão de duas bolsas de Concentrados de Hemácias dia 12/03/2019 e duas dia 01/04/2019.

Foram totalizados 84 dias de internação na UTI, gerando disfunções como fraqueza muscular generalizada sendo mensurado através da escala medical researchi council (MRC), rigidez articular de MMII, imobilismo, uso prolongado de VMI, desmame difícil e pneumonia. Foram traçadas metas diárias e uso de protocolos fisioterapêuticos, como o de mobilização precoce, com exercícios de fortalecimento muscular global, uso do cicloergômetro, sedestação, alongamentos, exercícios passivos/ativos, mobilização articular (Quadro 1 e 2).

Além da parte motora foi intensificada também a fisioterapia respiratória, pois a paciente apresentava grandes distúrbios funcionais devido muito tempo com via aérea artificial ocasionando desmame difícil. Foram aplicadas manobras de reexpansão pulmonar, técnicas de higiene brônquica e treinamento de endurance para musculatura respiratória. Os atendimentos fisioterapêuticos, na UTI, eram realizados diariamente no período da manhã, e em alguns dias à tarde, com a duração mínima de uma hora.

O desmame ventilatório iniciou-se com a estabilização do quadro da paciente submetendo a mesma a modalidade de ventilação por pressão de suporte (PSV). Com a adaptação da paciente a este modo passouse então a fazer os testes de respiração espontânea (TRE) no modo ventilatório Pressão de Suporte (PSV) com pressão de $7 \mathrm{cmH} 2 \mathrm{O}$ e PEEP de $5 \mathrm{cmH} 2 \mathrm{O}$. No dia 16 de abril, após 66 dias sob VM, a paciente foi submetida à retirada da máquina ventilatória em períodos intercalados do dia e ficando com suporte de $\mathrm{O} 2$ através de Venturi inicialmente com fluxo de $50 \%$ de $\mathrm{O} 2$ sendo reduzida gradativamente até a paciente não necessitar mais de suporte de oxigênio.

Após 60 dias de internação a paciente apresentou quadro de irritação, apatia e não estava colaborativa para realização da fisioterapia, sendo assim foram traçados planos terapêuticos juntamente com a equipe multidisciplinar onde incluiu passeios em área aberta no hospital, onde a paciente era levada com os dispositivos de monitorização em seu próprio leito. Essa estratégia gerou excelentes resultados, pois, a paciente ficou motivada para continuar o tratamento, visto que nessa fase a colaboração da paciente é de extrema importância. Assim, a cada evolução ela era levada a um passeio externo. Após passar longos períodos fora da VMI e o seu quadro ter se estabilizado a paciente teve alta da UTI. Em 06/05/19 foi transferida para a enfermaria. Continuava em uso de TQT, porém em respiração espontânea e ar ambiente.

$\mathrm{Na}$ enfermaria a paciente recebia atendimento fisioterapêutico $1 \mathrm{vez}$ ao dia (de segunda a sexta) e perante avaliação de força muscular registrada no prontuário a paciente apresentava MRC de 12 . A meta fisioterapêutica estabelecida para a paciente foi de melhora de ADM e força muscular e consequentemente funcionalidades. Foram realizados exercícios ativos, ativo-assistido, sedestação beira leito, alongamento, mudança de decúbito e controle de tronco, além de acompanhamento com a fonoaudióloga e nutricionista.

Em 21/05/2019, foi iniciado o protocolo de decanulação juntamente com a fonoaudióloga que inclui testes de fala, voz, deglutição, entre outros. Após ter os testes do protocolo positivos foi realizado o procedimento sem intercorrências. Após três dias da decanulação houve melhora progressiva de força muscular global, melhora na ADM de músculos de membros inferiores (MMII), melhora na motricidade conseguindo então sentar sem apoio.

Foram contabilizados 8 dias de IOT e 90 dias de traqueostomia. O protocolo de desmame da VM durou cerca de dez dias e a decanulação evoluiu após 27 dias de desmame da VM. Dia 24/05/19 foi programada a alta da paciente para casa, com orientações fisioterapêutica e médica e com datas para retorno e acompanhamento ambulatorial. Totalizaram-se 102 dias desde a internação no hospital do município de origem até a alta hospitalar. 
Quadro 1 - Evolução semanal da paciente sendo detalhado o plano e a intervenção terapêutica aplicada em cada fase, juntamente com os resultados alcançados.

\begin{tabular}{|c|c|c|c|}
\hline Semanas & Alteração functional & $\begin{array}{l}\text { Intervenção } \\
\text { fisioterapêutica }\end{array}$ & Resultados alcançados \\
\hline $1^{a}$ semana & $\begin{array}{l}\text { Diplopia, disartria, taquidispnéia, } \\
\text { Imobilismo. }\end{array}$ & Plano terapêutico 1. & Manteve a mobilidade articular e funcionalidade pulmonar. \\
\hline $2^{\mathrm{a}}$ semana & Imobilismo diminuição de ADM. & Plano terapêutico 1. & $\begin{array}{c}\text { Ganho de mobilidade articular, alteração do modo ventilatório } \\
\text { (espontâneo). }\end{array}$ \\
\hline $3^{\mathrm{a}}$ semana & $\begin{array}{l}\text { Fraqueza muscular generalizada, } \\
\text { diminuição de ADM e hipomobilidade. }\end{array}$ & Plano terapêutico 1. & $\begin{array}{c}\text { Ganho de mobilidade articular, desmame ventilatório, ausência de } \\
\text { lesões por pressão. }\end{array}$ \\
\hline $4^{a}$ semana & $\begin{array}{l}\text { Fraqueza muscular generalizada, } \\
\text { diminuição de ADM e hipomobilidade. }\end{array}$ & $\begin{array}{l}\text { Plano terapêutico } 3 \text { e } 4 \text {. } \\
\text { Após piora hemodinâmica } \\
\text { retorno com plano } \\
\text { terapêutico } 1 \text { e } 2\end{array}$ & $\begin{array}{c}\text { Manteve-se a mobilidade articular, houve melhora da força muscular } \\
\text { em extremidades. Após piora hemodinâmica houve RNC e } \\
\text { diminuiçãao da força alcançada em extremidades. Segue em modo } \\
\text { ventilatório espontâneo. }\end{array}$ \\
\hline $5^{\mathrm{a}}$ semana & $\begin{array}{l}\text { Fraqueza muscular generalizada e } \\
\text { hipersecretividade diminuição de ADM. }\end{array}$ & Plano terapêutico 2 e 3. & $\begin{array}{l}\text { Manteve-se preservada a mobilidade articular, houve melhora de } \\
\text { força muscular e resposta a comandos verbais e melhora da ADM. } \\
\text { Segue em modo ventilatório espontâneo com bom drive respiratório. }\end{array}$ \\
\hline $6^{\mathrm{a}}$ semana & $\begin{array}{l}\text { Fraqueza muscular generalizada, } \\
\text { hipomobilidade. }\end{array}$ & Plano terapêutico 2 e 3. & Manteve-se mobilidade articular e ADM. \\
\hline $7^{a}$ semana & $\begin{array}{l}\text { Fraqueza muscular generalizada, } \\
\text { hipomobilidade. }\end{array}$ & Plano terapêutico 3 e 4. & $\begin{array}{l}\text { Mantém-se mobilidade articular e ADM. Apresenta melhora de força } \\
\text { muscular global mais acentuada no hemicorpo esquerdo. }\end{array}$ \\
\hline $8^{\underline{a}}$ semana & $\begin{array}{l}\text { Fraqueza muscular, hipomobilidade, } \\
\text { paralisia facial. }\end{array}$ & $\begin{array}{l}\text { Plano terapêutico } 4,5 \text {, IF } 1 \text { e } \\
\text { IF } 4 .\end{array}$ & $\begin{array}{c}\text { Apresentou melhora da força muscular global, melhora ao contactar } \\
\text { através de gestos e melhora das expressões faciais. }\end{array}$ \\
\hline $9^{a}$ semana & $\begin{array}{c}\text { Rigidez articular e diminuição de ADM em } \\
\text { ombro e MID, Hipomobilidade e fraqueza } \\
\text { muscular, paralisia facial. }\end{array}$ & $\begin{array}{l}\text { Plano terapêutico } 4,5 \text { e IF } 1 \text {, } \\
\text { IF } 2 \text {, IF } 4 \text { e IF } 5 .\end{array}$ & $\begin{array}{c}\text { Evoluiu com piora de ADM e apresentou rigidez articular mais } \\
\text { acentuada em MID, segue contactuante através de gestos e } \\
\text { expressões faciais e melhora de força muscular. }\end{array}$ \\
\hline
\end{tabular}




\begin{tabular}{|c|c|c|c|}
\hline Semanas & Alteração functional & $\begin{array}{c}\text { Intervenção } \\
\text { fisioterapêutica }\end{array}$ & Resultados alcançados \\
\hline $10^{\text {a }}$ semana & $\begin{array}{l}\text { Rigidez articular e diminuição de ADM em } \\
\text { MID. Hipomobilidade e fraqueza } \\
\text { muscular, paralisia facial. }\end{array}$ & $\begin{array}{l}\text { Plano terapêutico } 5,6 \text { e IF } 1 \text {, } \\
\text { IF } 2 \text {, IF } 4 \text { e IF } 5 .\end{array}$ & $\begin{array}{c}\text { Evoluiu com piora de ADM e apresentou rigidez articular mais } \\
\text { acentuada em MID. Contactuante através de gestos e expressões } \\
\text { faciais e melhora de força muscular. Iniciado o desmame da VMI } \\
\text { com retirada intermitente do ventilador. }\end{array}$ \\
\hline $11^{a}$ semana & $\begin{array}{l}\text { Rigidez articular e diminuição de ADM em } \\
\text { MID. Hipomobilidade e fraqueza } \\
\text { muscular, paralisia facial. }\end{array}$ & $\begin{array}{c}\text { Plano terapêutico } 5,6 \text { e IF } 1 \text {, } \\
\text { IF } 2 \text {, IF } 4 \text { e IF } 5 .\end{array}$ & $\begin{array}{c}\text { Mantém-se o quadro de rigidez articular e diminuição de ADM em } \\
\text { MID, segue com evolução de força muscular global e desmame da } \\
\text { VMI com retirada intermitente do ventilador. }\end{array}$ \\
\hline $12^{\mathrm{a}}$ semana & $\begin{array}{c}\text { Rigidez articular e diminuição de ADM em } \\
\text { MID. Hipomobilidade e fraqueza } \\
\text { muscular. }\end{array}$ & $\begin{array}{c}\text { Plano terapêutico } 5,6 \text { e IF } 2 \text {, } \\
\text { IF } 4 \text {, IF } 5 \text { e IF } 6 .\end{array}$ & $\begin{array}{l}\text { Obteve melhora da rigidez articular e ADM de MID. Mantém-se } \\
\text { contactuante através de gestos e expressões faciais e com melhora } \\
\text { da força muscular global. Evolui bem com o desmame da VMI e } \\
\text { iniciado testes com a fonoaudióloga para liberação de dieta VO. } \\
\text { Iniciado desmame do balonete mantendo-o desinsuflado. }\end{array}$ \\
\hline $13^{\mathrm{a}}$ semana & $\begin{array}{c}\text { Rigidez articular e diminuição de ADM em } \\
\text { MID. Hipomobilidade e fraqueza } \\
\text { muscular. }\end{array}$ & $\begin{array}{l}\text { Plano terapêutico } 5,6 \text { e IF } 2 \text {, } \\
\text { IF } 4 \text {, IF } 5 \text { e IF } 6 .\end{array}$ & $\begin{array}{c}\text { Obteve melhora da rigidez articular e ADM, melhora da força } \\
\text { muscular global. Segue em desmame do balonete e evolui de alta } \\
\text { para a enfermaria. }\end{array}$ \\
\hline $14^{\mathrm{a}}$ semana & $\begin{array}{c}\text { Rigidez articular e diminuição de ADM em } \\
\text { MID. Hipomobilidade e fraqueza } \\
\text { muscular. }\end{array}$ & $\begin{array}{l}\text { Plano terapêutico } 5,6,7 \text { e IF } \\
2 .\end{array}$ & $\begin{array}{c}\text { Apresenta melhora do quadro geral com boa evolução da força } \\
\text { muscular global. Segue em RE em AA. Ainda em acompanhamento } \\
\text { com a fonoaudióloga evoluiu o desmame do balonete, iniciado e } \\
\text { teste de voz e liberado a dieta VO. }\end{array}$ \\
\hline $15^{\mathrm{a}}$ semana & $\begin{array}{c}\text { Rigidez articular e diminuição de ADM em } \\
\text { MID. Hipomobilidade e fraqueza } \\
\text { muscular. }\end{array}$ & $\begin{array}{l}\text { Planos terapêuticos } 5,6 \text { e } 7 . \\
\text { IF 2, IF 4, IF } 6 \text { e IF } 7 .\end{array}$ & $\begin{array}{c}\text { Nesta última semana houve uma importante evolução com a } \\
\text { melhora da rigidez articular e de força muscular periférica, realizado } \\
\text { sedestação à beira leito e fora do leito e ortostatismo. Segue em RE } \\
\text { em AA, com balonete desinsuflado e iniciado dieta VO. Realizado } \\
\text { decanulação e evolução de alta para casa. }\end{array}$ \\
\hline
\end{tabular}

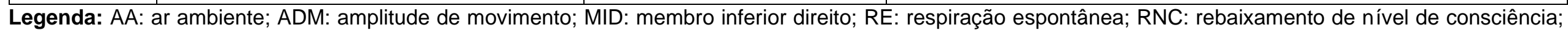

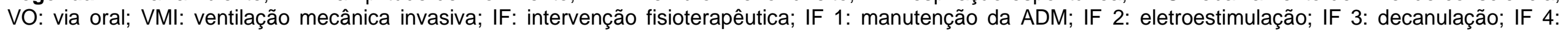
dissociação de cintura escapular e pélvica; IF 5: mímica facial; IF 6: crioterapia; IF 7: desmame da traqueostomia. Fonte: Silva TE, et al., 2020 
Quadro 2 - Planos terapêuticos aplicados de acordo com a fase de evolução da paciente.

\begin{tabular}{|c|c|}
\hline Plano terapêutico 1 & $\begin{array}{l}\text { HB, ajuste ventilatório para correção gasométrica, alongamento, } \\
\text { mobilização passiva e mudança de decúbito. }\end{array}$ \\
\hline Plano terapêutico 2 & $\begin{array}{c}\text { HB, desmame ventilatório, alongamento, mobilização passiva, ativo } \\
\text { assistida e mudança de decúbito. }\end{array}$ \\
\hline Plano terapêutico 3 & $\begin{array}{c}\mathrm{HB} \text {, desmame ventilatório, alongamento, exercícios ativos assistido, ativo } \\
\text { e mudança de decúbito. }\end{array}$ \\
\hline Plano terapêutico 4 & $\begin{array}{c}\text { HB, desmame ventilatório, alongamento exercícios ativo assistido, ativo, } \\
\text { mudança de decúbito e sedestação no leito. }\end{array}$ \\
\hline Plano terapêutico 5 & $\begin{array}{l}\text { HB, desmame ventilatório, alongamento exercícios ativo resistido, } \\
\text { mudança de decúbito, sedestação no leito e beira leito. }\end{array}$ \\
\hline Plano terapêutico 6 & $\begin{array}{c}\text { HB, desmame ventilatório e retirada da VMI, desmame de O2 } \\
\text { suplementar, alongamento, exercícios ativos resistido, sedestação no } \\
\text { leito e beira leito. }\end{array}$ \\
\hline Plano terapêutico 7 & $\begin{array}{l}\text { HB, desmame ventilatório e retirada da VMI, desmame de O2 } \\
\text { suplementar, alongamento, exercícios ativos resistido, sedestação no } \\
\text { leito, beira leito e ortostatismo. }\end{array}$ \\
\hline
\end{tabular}

Legenda: HB: higiêne brônquica; VMI: ventilação mecânica invasiva. Fonte: Silva TE, et al., 2020.

\section{DISCUSSÃO}

O botulismo alimentar é proveniente do consumo de alimentos contaminados pelo clostridium botulinum, toxina presente em alimentos geralmente relacionados com conservas caseiras de carnes, frutas e vegetais onde esses produtos são mal manuseados ou armazenados (CERESER ND, et al., 2008). Segundo a literatura o soro anti-botulismo deve ser administrado de forma mais precoce possível para eliminar a toxina ainda circulante no SNC e com isso uma evolução mais amena e melhor prognóstico da doença (SANTOS JS, et al., 2019; TORNESE M, et al., 2008).

Durante o desmame da sedação realizada pelo médico, é atribuição do fisioterapeuta avaliar a função neuromuscular do paciente utilizando como recurso a escala MRC para quantificar o grau de força muscular periférica com pontuação de 0 a 60 , sendo como regra quanto maior a pontuação maior a força muscular. Através desta escala é possível prescrever exercícios, traçar metas e condutas a serem desenvolvidas durante $o$ atendimento com o paciente (FELICIANO V, et al., 2019).

A síndrome do imobilismo é um conjunto de alterações que afetam vários sistemas do corpo, porém o mais predominante é o sistema osteomuscular levando a limitações funcionais do paciente acometido. É classificado como repouso prolongado no leito o período de 7 há 10 dias, sendo o imobilismo considerado acima de 12 dias e decúbito de longa duração acima de 15 dias. Ao final de quatro semanas o indivíduo perde cerca de $50 \%$ da sua força muscular inicial e essa permanência no leito causa diminuição da capacidade de realizar exercícios, descondicionamento físico e comprometimento de outros sistemas do corpo humano (CINTRA MMM, et al., 2013).

A mobilização precoce (MP) é um recurso terapêutico utilizado durante o período de internação para combater a síndrome do imobilismo, reduzindo as complicações decorrentes do repouso prolongado, diminuindo o tempo de VM, aumentando a força muscular mantendo e/ou recuperando o grau prévio de funcionalidade do paciente (SILVA APP, et al., 2010). A MP é uma terapêutica segura e eficaz que deve ser utilizada respeitando os critérios de segurança e/ou contraindicações, no intuito de diminuir o tempo de internação hospitalar, aumentar a sobrevida e a qualidade de vida pós-alta hospitalar (SILVA APP, et al., 2010; GOSSELINK R, et al., 2008; CHOI J, et al., 2008).

A cinesioterapia consiste em um conjunto de exercícios terapêuticos que auxiliam na reabilitação revertendo a limitação articular e muscular, através do fortalecimento e alongamento dos músculos, sendo utilizado em conjunto com os recursos terapêuticos manuais promove também redução do edema, aumento da ADM, mobilidade articular e força muscular global (DE AMORIM JSC, et al., 2014).

A neuroestimulação elétrica transcutânea (TENS) é um equipamento que produz correntes elétricas pulsadas baseadas na teoria das comportas da dor. É um método fisioterapêutico que pode ser utilizado no 
tratamento de dor aguda ou crônica a fim de promover uma melhora do controle da dor. Consiste na aplicação de impulsos elétricos no local a ser tratado com objetivo de ativar o sistema nervoso para exercer uma ação analgésica, ajudando no combate da dor (DE AMORIM JSC, et al., 2014).

A paralisia facial simétrica é uma disfunção que causa perda ou diminuição dos movimentos da face, pode ser causada pelo botulismo, sendo necessário intervenção fisioterapêutica. $O$ tratamento visa restabelecer a força e o trofismo muscular possibilitando o retorno funcional, diminuindo o problema na alimentação, fala e interação social. Os principais recursos fisioterapêuticos aplicados são cinesioterapia, massoterapia e exercícios de mímica facial. (GARANHANI MR, et al., 2007; BAUGH RF, et al., 2013).

Diante das alterações funcionais decorrentes do imobilismo a qual a paciente apresentou o tratamento fisioterapêutico foi de suma importância. Pois teve como objetivo evitar a incidência de fatores adversos decorrentes do imobilismo prolongado no leito, pois apresentou resultados favoráveis na prevenção da fraqueza muscular, diminuindo o tempo de VM e internação hospitalar, mantendo o grau prévio de funcionalidade do indivíduo e por fim melhorando a qualidade de vida pós alta hospitalar.

\section{REFERÊNCIAS}

1. BARBOZA MMO, et al. Surto familiar de botulismo no estado do Ceará: relato de caso. Revista da Sociedade Brasileira de Medicina Tropical, 2011; 44(3): 400-402.

2. BAUGH RF, et al. Clinical practice guideline: Bell's palsy. Otolaryngology-Head and Neck Surgery, 2013; 149(3_supl): S1-S27.

3. BRASIL. Ministério da Saúde (MS). Gabinete do Ministro. Portaria n² 2472 de 31 de agosto de 2010. Brasília: DF; 2010. Disponível em: http://bvsms.saude.gov.br/bvs/saudelegis/gm/2010/prt2472_31_08_2010.html.

4. BRASIL. Ministério da Saúde (MS). Guia de Vigilância em Saúde [recurso eletrônico]. Brasília: DF; 2017. Disponível em: https://bvsms.saude.gov.br/bvs/publicacoes/guia_vigilancia_saude_3ed.pdf.

5. BRASIL. Ministério da Saúde (MS). Manual Integradō de vigilância epidemiológica do botulismo. Brasília: DF; 2006. Disponível

http://bvsms.saude.gov.br/bvs/publicacoes/manual_integrado_vigilancia_epidemiologica_botulismo.pdf.

6. BRASIL. Ministério da Saúde (MS). Manual integrado de vigilância, prevenção e controle de doenças transmitidas por alimentos. $\quad$ Brasília: $\quad$ DF; $2010 . \quad$ Disponível em: http://bvsms.saude.gov.br/bvs/publicacoes/manual_integrado_vigilancia_doencas_alimentos.pdf.

7. CARDOSO T, et al. Botulismo de origem alimentar: revisão de cinco casos. Acta Médica Portuguesa, 2004; 17(1): 54-8.

8. CERESER ND, et al. Botulismo de origem alimentar: Revisão bibliográfica. Ciência Rural, Santa Maria, 2008; 38(1): 208-287.

9. $\mathrm{CHOl} \mathrm{J}$, et al. Mobility interventions to improve outcomes in patients undergoing prolonged mechanical ventilation: a review of the literature. Biological research for nursing, 2008; 10(1): 21-33.

1. CINTRA MMM, et al. Influência da fisioterapia na síndrome do imobilismo. In Colloquium Vitae. ISSN: $1984-6436$. 2013; 5(1): pp. 68-76.

10. DE AMORIM JSC, et al. Efeitos da terapia manual e eletroterapia na osteoartrite de joelho. ConScientiae Saúde, 2014; 13(1): 11-20.

11. FELICIANO $\mathrm{V}$, et al. A influência da mobilização precoce no tempo de internamento na Unidade de Terapia Intensiva. Cardiopulmonary and Critical Care Physiotherapy, 2019; 3(2): 31-42.

12. FIGUEIREDO MAA, et al. Considerações acerca de dois casos de botulismo ocorridos no Estado da Bahia. Revista da Sociedade Brasileira de Medicina Tropical, 2006; 39(3): 289-291.

13. GARANHANI MR, et al. Fisioterapia na paralisia facial periférica: estudo retrospectivo. Revista Brasileira de Otorrinolaringologia, 2007; 3(1): 112-115.

14. GIL AC. Como elaborar projetos de pesquisa. São Paulo: Atlas, 2002; 4: 175.

15. GOSSELINK R, et al. Physiotherapy for adult patients with critical illness: recommendations of the European Respiratory Society and European Society of Intensive Care Medicine Task Force on physiotherapy for critically ill patients. Intensive care medicine, 2008; 34(7): 1188-1199.

16. MONTEZUMA Cruz. 2019. In: Secom - Governo de Rondônia. Rondônia: Portal do Governo do Estado de Rondônia. Disponivel em: <http://www.rondonia.ro.gov.br/agevisa-envia-soro-para-vitimas-de-botulismo-em-sao-miguel-ealerta-para-o-cuidado-com-procedencia-e-manuseio-de-alimentos/>. Acesso em: 15 Mai. 2020.

17. SANTOS JS, et al. Epidemiologia e fatores de riscos relacionados à intoxicação alimentar causada por Clostridium Botulinum: Uma revisão narrativa. Clinical \& Biomedical Research, 2019; 39(2).

18. SILVA APP, et al. Efeitos da fisioterapia motora em pacientes críticos: revisão de literatura. Revista Brasileira de Terapia Intensiva, 2010; 22(1): 85-91.

19. TORNESE M, et al. Epidemiologia e fatores de risco associados ao botulismo alimentar e ao botulismo infantil: onde e quando? Rev. chil. infectol. Santiago, 2008; 25(1): 22-27.

20. ZATTI CA. Botulismo: Conhecendo os casos brasileiros notificados entre 2007 a 2013. Revista Contexto \& Saúde, 2013; 13(24-25): 21-26. 